\title{
SPECIAL 6-COMPONENT JET RIG BALANCE FOR STUDYING NEW THRUST VECTORING CONCEPTS
}

\author{
M. A. Ramaswamy \\ Professor, Department of Aerospace Engineering \\ Indian Institute of Science, Bangalore, INDIA
}

F. S. Alvi

Assistant Professor, Department of Mechanical Engineering

FAMU-FSU College of Engineering, Tallahassee, Florida, USA

\author{
A. Krothapalli \\ Don Fuqua Professor \& Chairman, Department of Mechanical Engineering \\ FAMU-FSU College of Engineering, Tallahassee, Florida, USA
}

\begin{abstract}
A new concept for efficient thrust vectoring of jet exhausts for aerospace applications, known as CounterFlow Thrust Vector (CFTVC) Control has been investigated at the Fluid Mechanics Research Laboratory (FMRL) over the past few years. Although pressure measurements and flow visualization results have proven the efficacy of this technique, it was felt that more direct measurements of the vectoring angles and thrust efficiency are needed to evaluate the CFTVC performance more rigorously. Therefore a special 6component balance to measure the main thrust as well as the lateral thrust, (which may be in any direction) was conceived, designed and fabricated at FMRL. This paper provides complete details of the design, fabrication, assembly, calibration and data reduction procedures of this balance. Some typical results obtained using this rig are also presented which clearly demonstrate the accuracy and usefulness of this instrument.
\end{abstract}

\section{INTRODUCTION}

In recent years a unique fluidic-based technique, known as CounterFlow Thrust Vector Control (CFTVC), for efficient thrust vectoring of jet exhausts for aerospace applications, has been investigated at the Fluid Mechanics Research Laboratory (FMRL). These studies [1,2 \& 3] have revealed that thrust vectoring can be achieved by establishing a secondary counterflow stream along a selected, streamwise segment of the outer surface of the jet shear layer.

Fig. 1 depicts an abbreviated side-view of the CFTVC nozzle-collar assembly. The figure also indicates the conditions necessary to achieve jet vectoring at an angle $\delta_{v}$ due to the secondary counterflowing stream along the upper shear layer. This technique has been successfully demonstrated using nozzles of different geometries, e.g. axisymmetric, diamond-shaped and rectangular. The primary air which can be heated up to $800^{\circ} \mathrm{F}$ exhausts through the nozzle and an appropriately designed collar (dependent upon the nozzle shape) is placed around the primary jet nozzle creating gaps between the nozzle and collar surface through which counterflow can be applied. While the rectangular jet can only be vectored up and down in a single plane, the diamond and round jets can be vectored in multiple planes. Hence the rectangular jet arrangement is commonly referred to as a single-axis or pitch vectoring system while the diamond and round jet systems are called multi-axis systems. The interested reader is directed to references $1-3$ for further details.

A typical Planar Laser Scattering (PLS) image of a vectored Mach 2 axisymmetric jet is shown in Fig. 2, clearly illustrating the efficacy of the CFTVC system. In addition to visual data it was important to obtain direct force measurements documenting the effectiveness of this fluidic control concept. Therefore, a specialized 6component balance to measure the main thrust as well as the lateral thrust (which may be in any direction) was conceived, designed and fabricated at FMRL.

In this paper details of the design concept, expected design loads, actual design and fabrication details, calibration details and data reduction procedure will be discussed. Also, some typical results obtained using this balance will be presented.

\section{CONCEPTUAL DESIGN}

The schematic arrangement for a 3-component balance to illustrate the basic design concept is shown in Fig. 3. As shown, the supply line and the vacuum lines must be isolated from the metric part through very flexible hoses 
or bellows. The small amount of elastic constraint offered by the bellows is taken into account in the calibration. For the sake of flexibility in the cold flow case, and as a matter of necessity in the hot flow case, the forces acting on the metric part of the jet rig are transferred to an outer tube, which forms part of the balance. The arrangement shown in Fig. 3 corresponds to the cold case. For hot flow experiments, some modifications are necessary to prevent large thermal stresses from being introduced in the inner and outer tubes.

The conceptual design of the 6-component balance is illustrated in Fig. 4. The outer tube, on which the forces are to be measured is supported by 6 links (which are rigid in the axial direction) through load cells as shown. The reference $\mathrm{x}$ axis for the balance is the tube axis. The y $\& \mathrm{z}$ axes are as indicated. The clockwise moments about these axes are denoted by $P, Q$ and $R$ respectively. The forces measured by the load cells are denoted by $V I$, $V 2, V 3, H 1, H 2$ and $H 3$ (see Fig. 4). The forces $X, Y, Z$ and the moments $P, Q, R$ acting on the tube in terms of the forces measured by the load cells are given by equation 1 below. Compressive loads on the load cells are considered positive.

$$
\begin{aligned}
& \mathrm{X}=\mathrm{H} 1+\mathrm{H} 2 \\
& \mathrm{Y}=-\mathrm{H} 3 \\
& \mathrm{Z}=\mathrm{V} 1+\mathrm{V} 2+\mathrm{V} 3 \\
& \mathrm{P}=\mathrm{L} 2(\mathrm{~V} 2-\mathrm{V} 3) \\
& \mathrm{Q}=-(\mathrm{L} 1)(\mathrm{V} 1) \\
& \mathrm{R}=\mathrm{L} 2(\mathrm{H} 2-\mathrm{H} 1)
\end{aligned}
$$

The balance should be fabricated with extreme precision so that the linkages are truly vertical or horizontal and orthogonal, and the lengths L1 \& L2 are known precisely. In an ideal case, if any particular load, say V1 is applied then the output in the other load cells should be zero. However, since the linkages have some - though very small - constraint in the lateral direction very minor inaccuracies will always be present. Consequently, even if the balance is carefully fabricated, there will always be some interaction outputs in the other load cells. The design should aim at keeping these interactions as small as possible. However, as we will discuss later, even these small interactions are taken into consideration in the calibration.

The forces acting on the jet rig and their locations can be found as follows:

Thrust $\mathrm{T}$ along $\mathrm{x}$ axis $=\mathrm{X}$

Lateral force $F_{y}$ along $y$ axis $=Y$

Lateral force $F_{z}$ along $\mathrm{z}$ axis $=Z$

Location of $F_{y}, d_{1}=-R / Y$

(2c)

(2d)

$$
\text { Location of } F_{z}, d_{2}=Q / Z
$$

and the radius $r$ at which the resultant of $F_{y}$ and $F_{z}$ acts is given by:

$$
r=\frac{P}{\sqrt{F_{y}^{2}+F_{z}^{2}}}=\sqrt{y^{2}+z^{2}}
$$

where $y=k r \sin \theta$ and $z=-k r \cos \theta$, where $k=+1$ for $P$ $+v e$ (positive) and -1 for $P$-ve and $\theta=\tan ^{-1}\left(F_{z} / F_{y}\right)$. The proper value of $\theta$ to be used is given below.

$$
\begin{array}{ll}
0 \leq \theta \leq \pi / 2 & F_{y} \text { and } F_{z} \text { are }+v e \\
\pi / 2<\theta \leq \pi & F_{y} \text { is }-v e \text { and } F_{z} \text { is +ve } \\
\pi<\theta \leq 3 \pi / 2 & F_{y} \text { and } F_{z} \text { are -ve } \\
3 \pi / 2<\theta \leq 2 \pi & F_{y}+v e \text { and } F_{z}-v e
\end{array}
$$

In this application of thrust vectoring, the rolling moment $P$ is expected to be zero and distances $d_{1}$ and $d_{2}$ are expected to be equal. However the equations above consider the general case. Note that if $F_{y}$ or $F_{z}$ is zero then, $d_{1}$ or $d_{2}$ becomes infinity. This means that the particular lateral force is zero and the off-axis thrust gives rise to $\mathrm{Q}$ or $\mathrm{R}$ where the off-axis distances are given by:

$$
y=-R / T \text { and } z=Q / T
$$

\section{Load Capacity}

Based upon the nozzle geometry and Mach number, it was estimated that the maximum thrust for nozzles tested in the facility would not exceed $120 \mathrm{lbf}$. Assuming that the maximum jet thrust vectoring angle would be $30^{\circ}$, the lateral load (which could be in any direction) was estimated to be $60 \mathrm{lbf}$. A preliminary layout of the balance taking into consideration the space available resulted in length $\mathrm{L} 1=18$ inches and $\mathrm{L} 2=9$ inches. An approximate estimate of the weight of tube support, air supply tube, plenum chamber etc., was around $80 \mathrm{lbs}$ and the C.G. of the rig was estimated to be 8 inches downstream of the balance reference origin. Taking into consideration direct and lateral thrust and the weight of the system, and allowing for a margin of error, the capacity of the load cells V1, V2, V3, H1 and H2 was chosen to be $200 \mathrm{lbf}$. and that of $\mathrm{H} 3$ to be $100 \mathrm{lbf}^{1}$

\section{DETAILS OF THE BALANCE}

The isometric sketch, drawings showing three orthogonal projections and a photograph of the complete rig are shown in Figs. 5, 6 and 7 respectively. Details of the

\footnotetext{
${ }^{1}$ Model 1500 ASK load cells manufactured by Interface ${ }^{T M}$ Inc.
} 
various aspects of the balance will now be discussed in subsequent sections.

\section{Structure Details \\ Structural details of the balance will be reviewed in this section; the reader is advised to refer to Figs. 5 and 6 to better follow this discussion. The balance consists of a basic bottom structure made up of 3 in. $x$ in. aluminum sections (formed by welding 3 in. $\times 1.5$ in. channels ) upon which the front and rear frames are mounted. A triangular structure is mounted on top spanning the front and rear frames as shown in Fig. 5. All of these frames are also made up of $3 \times 1.5$ inch aluminum channels. Dowels are provided to permit precision assembly of the parts.}

As previously mentioned, there is an outer (steel) tube to which all the forces acting on the jet rig are transferred. Two arms, made of 2 in. pipe, with end flanges welded to this outer tube are welded near the front of the outer tube. Rectangular blocks, equipped with precisely machined keyways to allow for accurate alignment of the outer tube, are attached to these flanges. Henceforth, this entire assembly - outer tube, 2 in. pipes and flanges - will be referred to as the 'outer tube'. This outer tube is supported by three vertical links V1, V2 and V3 hanging from the top frame, two horizontal links $\mathrm{H} 1$ and $\mathrm{H} 2$ attached to the rear frame, and another horizontal link $\mathrm{H} 3$ attached to the vertical column on the bottom frame.

The most critical aspects of the fabrication of the balance is that when assembled, the vertical links should be absolutely vertical and the horizontal links should be absolutely horizontal; the links $\mathrm{H} 1$ and $\mathrm{H} 2$ should be absolutely parallel to each other and both of them being perpendicular to links H3. Furthermore, H3 should be in the plane of the links V2 and V3. The degree of accuracy desired dictates that during assembly of these links, the misalignment should be not more than $0.5 \mathrm{~mm}$. (0.02inch). The need of this accuracy is due to the fact that these links are designed to be very flexible in the lateral direction but are strong enough to take the axial compression load without buckling. If there is significant misalignment, then these links would be subjected to eccentric loading and may fail, as they are not be able to take large bending moments. It is important to note that the load cell attached to the frames at the ends of these links have a maximum deflection of about 0.003 inch under maximum loading. Therefore, the linkages are not subjected to any appreciable lateral deflection due to thrust loads on the balance. The bending load on the links arises mainly due to misalignment and hence they should be avoided as much as possible. The accuracy requirements are indicated in the 3-view drawing shown in Fig. 6. The precautions taken during fabrication to achieve this goal are described in detail in reference 4 .

The manner in which the loads acting on the jet rig are transferred to the outer tube is also illustrated in Fig. 3. for the cold case. The inner tube consists of a 2.5 inch nominal stainless steel tube which is threaded at both ends to which the supply line and the nozzle with the thrust vectoring collar are attached. As shown in Fig. 3 two split -rings or collars are clamped to the inner tube, the rings are held in place by bolts through the outer tube thus transferring the loads to the outer tube. More details of this aspect of the balance are provided in a later section on the inner tube assembly.

Provision is made to calibrate the balance using dead weights. Attachment points are provided in the outer tube (Figs. $5 \& 6$ ) to enable application of load V1, V2, V3, $\mathrm{H} 1, \mathrm{H} 2$ and H3. For applying horizontal loads the cable has to pass over a pulley. For the sake of simplicity in the design and more importantly in the fabrication process, only tension loads can be applied during calibration.

\section{Design of the links}

The design of the links is critical in that they transmit the forces from the outer tube to the load cells; some details of the link design are given here. The primary requirement for the links is that while being as flexible as possible in the lateral direction they should also be capable of withstanding large axial loads $(200 \mathrm{lbf})$ without buckling. It is for this reason that, instead of having a long flexure, the link has two thin flexures as shown in Fig. 8. Since the links form parallelograms of linkages, the link axis at the deflected end remains parallel to the link axis at the fixed end. A detailed drawing of the shortest link, which has an overall length of 8 in., is provided in Fig. 8. For this flexure, the lateral deflection to load ratio, $\mathrm{d} / \mathrm{P}$, is $0.035 \mathrm{inch} / \mathrm{lb}$ while the axial deflection of the load cells at maximum loads is $0.003 \mathrm{in}$. for both 100 and $200 \mathrm{lb}$ load cells. Even if one conservatively considers the more flexible case, i.e. the $100 \mathrm{lb}$ load cell, the lateral flexibility of the link is more then 1000 times the axial flexibility of the load cell. Furthermore, the axial flexibility of the link itself is of the order or 0.002 inch for a $200 \mathrm{lbf}$ load, even if this is taken into consideration, the lateral flexibility is of the order of 500 times the axial flexibility. This is considered to be quite satisfactory for our purpose.

The thin flexure is subjected to a stress of $16500 \mathrm{psi}$ for a $200 \mathrm{lb}$. direct load. The bending stress due to lateral eccentricity of 0.02 inch is about 8000 psi. For an eccentricity of 0.02 inch, if an axial load of $200 \mathrm{lb}$ acts upon the link, an additional bending stress of about 24000 
psi. would arise. Therefore, the link is made of special alloy steel with a UTS of 160,000 psi. Nevertheless, considerable care should always be taken in handling the flexure.

\section{Jet rig inner tube assembly}

As shown in Fig. 3, the jet rig inner assembly consists of 3 parts: the central inner stainless tube with a nominal diameter of $2.5 \mathrm{in}$.; the downstream nozzle system which includes the supersonic nozzle and the associated counterflow/suction collar and; the upstream entry duct which supplies air to the nozzle through the inner tube. Air is delivered to the entry duct through four $1.5 \mathrm{in}$. pipes orthogonal to the duct and arranged symmetrically around its periphery. Air to these pipes is in turn delivered by very flexible hoses or bellows. The flexible tubes are used to isolate the balance from the upstream air supply thereby preventing any forces from being transmitted to the balance. The picture of the assembled rig, shown in Fig. 7, together with the schematic in Fig. 3 should help to further clarify this arrangement (Note that the nozzle and counterflow assembly is not attached to the downstream end of the inner tube in the image shown in Fig. 7).

Two split rings or collars are clamped around the central inner tube and can be placed at any desired longitudinal position. These collars fit snugly inside the outer tube and are held firmly by bolts in the outer tube. The nozzle system and the entry duct have to be assembled after the central inner tube has been fixed in the outer tube. A very careful assembly procedure was evolved [4] to ensure that the required accuracy was attained under final assembled condition and to also ensure that the flexible links were not subjected to undue bending loads during assembly.

\section{CALIBRATION}

The purpose of the calibration is to find the relationship between a force experienced by the balance to the output (usually measured in volts) of the six load cells. From our earlier discussion we know that the net force acting on the balance is the vector sum of forces $\mathrm{X}, \mathrm{Y}$ and $\mathrm{Z}$. In turn, these are related to the forces measured by the six cells $(\mathrm{H} 1, \mathrm{H} 2$, etc., see equation 1$)$. If we define the output voltages from the six load cells as a $6 \times 1$ matrix called $O$ and the forces acting on the six cells as another 6 xl matrix called $F$, then the two can related by the following:

$$
[\mathbf{O}]=[\mathbf{C}][\mathbf{F}]
$$

where $C$ is a $6 \times 6$ coefficient matrix obtained through the calibration. In expanded form equation 6 can be written as follows:

$\left[\begin{array}{l}\mathrm{O}_{v 1} \\ \mathrm{O}_{v 2} \\ \mathrm{O}_{v 3} \\ \mathrm{O}_{h 1} \\ \mathrm{O}_{h 2} \\ \mathrm{O}_{h 3}\end{array}\right]=\left[\begin{array}{llllll}C_{v 1 v 1} & C_{v 1 v 2} & C_{v 1 v 3} & C_{v 1 v h 1} & C_{v 1 h 2} & C_{v 1 h 3} \\ C_{v 2 v 1} & C_{v 2 v 2} & C_{v 2 v 3} & C_{v 2 h 1} & C_{v 2 h 2} & C_{v 2 h 3} \\ C_{v 3 v 1} & C_{v 3 v 2} & C_{v 3 v 3} & C_{v 3 h 1} & C_{v 3 h 2} & C_{v 3 h 3} \\ C_{h 1 v 1} & C_{h 1 v 2} & C_{h 1 v 3} & C_{h 1 h 1} & C_{h 1 h 2} & C_{h 1 h 3} \\ C_{h 2 v 1} & C_{h 2 v 2} & C_{h 2 v 3} & C_{h 2 h 1} & C_{h 2 h 2} & C_{h 2 h 3} \\ C_{h 3 v 1} & C_{h 3 v 2} & C_{h 3 v 3} & C_{h 3 h 1} & C_{h 3 h 2} & C_{h 3 h 3}\end{array}\right]\left[\begin{array}{c}V 1 \\ V 2 \\ V 3 \\ H 1 \\ H 2 \\ H 3\end{array}\right]$

In the above matrix, each of the terms in matrix $F$ corresponds to the axial force experienced by each link. Similarly, each of the terms in matrix $O$ corresponds to the voltage measured by a particular load cell due to a force applied to the balance. For example, $O_{v 1}$ is the voltage output of load cell connected to link V1 while $\mathrm{O}_{\mathrm{hl}}$ is the output from load cell $\mathrm{H} 1$ and so on. The significance of the terms in the coefficient matrix , C, will become clear from the ensuing discussion of the calibration procedure.

\section{Calibration Procedure}

During calibration an excitation voltage (10 volts in the present case) is applied to all the load cells and the no load outputs from all six load cells is recorded. Then, using precision dead weights, pure axial loads, are applied along each link in incremental steps spanning the entire load range. This procedure is repeated for all the links. The sensitivity coefficients, represented by the terms in matrix $C$, of each of the load cells are then determined using a linear least square fit [4].

Note that for each load component say V2, there are 6 coefficients, viz. $\mathrm{C}_{\mathrm{vlv2}}, \mathrm{C}_{\mathrm{v} 2 \mathrm{v} 2}, \mathrm{C}_{\mathrm{v} 3 \mathrm{v} 2}, \mathrm{C}_{\mathrm{h} 1 \mathrm{v} 2}, \mathrm{C}_{\mathrm{h} 2 \mathrm{v} 2}$ and $\mathrm{C}_{\mathrm{h} 3 \mathrm{v} 2}$ where the first subscript corresponds to the load cell whose output is being considered and the second subscript refers to the load that is being applied. In an ideal balance, only the direct coefficient $\mathrm{C}_{\mathrm{v} 2 \mathrm{v} 2}$, should exist and the other five interaction coefficients should be zero. (Note that all the off-diagonal coefficients are the interaction terms). Although any practical balance will always have interaction terms, in a well-designed balance the interaction coefficients should be less then $5 \%$ of the direct coefficient. For the present balance the largest interaction term was less than $3 \%$ of the direct coefficient.

The presence of interaction terms is not a serious problem as long as they are linear and repeatable. The accuracy of the balance is generally checked by applying some typical combination of loads. Then, by recording the load cell outputs, the loads are calculated and compared with the actual applied loads. The difference between these two 
values as a percentage of the applied load gives the accuracy of the balance. For the present balance, the maximum error between the work back loads and applied loads was well below $3 \%$.

It is worth restating that in order to obtain accurate measurements, the calibration must be performed very deliberately and carefully. For example, the weights of the calibration weights must be known precisely and if the dead weights are being suspended, as was done in the present case, one must ensure that they are aligned with the link axis and are not swinging. Once a calibration has been carefully performed and the coefficient matrix has been determined then any force (or a component in any direction) or net load acting on the rig can be determined by using equations 1 and 2 and the inverse of equation six as given below:

$[\mathrm{F}]=[\mathrm{C}]^{-1}[\mathbf{O}]$

where $[C]^{-1}$ is simply the inverse of the $C$ matrix.

Despite the use of flexible hoses and numerous measures taken to isolate the rig from the air supply, it was found that some forces were still transmitted through the air supply to the balance. This was primarily due to the fact that the pipes are stressed as they are pressurized during the actual tests, whereas the calibration is performed on the non-pressurized pipes. To account for this, a tare measurement was first taken by capping off the end of the nozzle and pressurizing the entire piping system to the stagnation pressures at which the jet is operated. These 'pressure loads' are then subtracted from the measurements made during the actual tests. Another way to fix this problem would be to perform the calibration while the pipes are pressurized. However since the calibration would have to be performed at all the operating pressures, this can be a very time-consuming process. As the results discussed in the next section demonstrate, after carefully calibrating the balance and using the tare measurements, very accurate results can be obtained with the present balance.

\section{RESULTS}

As stated in the introduction, the motivation behind the design of this balance was to gain the ability to directly measure the performance of the counterflow thrust vectoring technique [1-3]. To this end, thrust vector measurements for an axisymmetric, Mach 2 jet were obtained using the present balance.

A summary of the thrust performance is provided in Fig. 9, which shows a plot of the jet vector angle, $\delta_{\mathrm{v}}$ (see. Fig. $1)$ as a function of the parameter $\left(\Delta \mathrm{P}_{\text {exit }} A_{\text {side }}\right) /\left(\rho_{1} U_{1}{ }^{2} A_{\text {jet }}\right)$.
This pressure parameter is essentially proportional to the amount of counterflow applied to the primary jet (see reference 3 for details). In addition to the axisymmetric measurements, represented by filled circles, data for rectangular (symbols in the hatched region) and diamondshaped Mach 2 jets are also shown for completeness.

While the thrust angles for the round jet were obtained from direct force measurements using the balance, the $\delta_{v}$ 's for the other two geometries were obtained using a variety of techniques such as pressure distributions on the counterflow collar surface and flow visualization [3]. The plot clearly shows that the axisymmetric jet displays an almost linear behavior, very similar to the trends observed for the other two geometries. This agreement between the vector angles obtained using the force balance (for the round jet) and those obtained via other techniques gives us confidence that the present rig is operating properly.

Although Fig. 9 shows that the vectoring performance of the single-axis (rectangular) geometry is slightly better than the round jet (as well as the diamond-shaped jet), this difference is not due to errors in the force measurements of the axisymmetric jet obtained using the balance. We believe that the primary reason for the differences in thrust vector performance is due to a noticeable leakage between adjacent chambers of the collar assemblies for the multi-axis arrangement. This was determined by monitoring the static pressure distributions in the chambers adjacent to the one with counterflow. Leakage effects indicate that counterflow is not completely isolated from other parts of the shear layer, resulting in a degradation of performance principally due to the transverse pressure gradient across the shear layer. The leakage was more significant in the diamond nozzle-collar assembly resulting in a slightly lower efficiency for the diamond jet. Although these three-dimensional effects will undoubtedly influence the collar performance, we believe that they can be minimized by improvements in collar design [3].

As a further test of the jet rig balance capability and accuracy, thrust produced by four different nozzles was also measured. These include a round Mach 1.8 nozzle, plus a round, a rectangular and a diamond shaped nozzle, each with the same exit area and a design Mach number of 2 . All the nozzles were operated at design, over and under-expanded conditions and the measured thrust was compared to thrust predicted using 1-D compressible flow relations. Excellent agreement was found between measured and predicted thrust values for the round and rectangular nozzles, especially at design conditions where the agreement was within $1 \%$. The greatest difference 
between the measured and predicted values was displayed by the diamond jet where, at design conditions, the measured thrust was almost $4 \%$ below the predicted value. This result is anticipated, since the round and rectangular nozzles are expected to have lower thrust loss due to friction relative to the diamond jet, thus providing better agreement with 1-D predictions which do not account for the frictional losses. In summary, both the vectored and the unvectored thrust measurements for various nozzles provide ample evidence of the accuracy and usefulness of this balance.

\section{ACKNOWLEDGMENTS}

The authors would like to acknowledge the help of Dr. Paul Strykowski, Mr. Edward Barber, Mr. Martin Garcia and Mr. Donnell Washington in conducting some of the experiments. Support for this research, provided by the Office of Naval Research and NASA HQ, is gratefully acknowledged.

\section{REFERENCES}

[1] Strykowski, P. J., Krothapalli, A. \& Forliti, D. J., "Counterflow Thrust Vectoring of Supersonic Jets", AIAA Journal Vol. 34, No. 11, 1996, pp. 2306-2314.

[2] Washingtion, D. M., Alvi, F. S., Strykowski, P. J. \& Krothapalli, A., "Multiaxis Fluidic Thrust Vector Control of a Supersonic Jet using Counterflow", AIAA Journal, Vol. 34, No. 8, 1996, pp. 1734-1736.

[3] Alvi, F. S., Strykowski, P. J., Washingtion, D. M., \& Krothapalli, A., "Multiaxis Fluidic Thrust Vectoring of Supersonic Jets via Counterflow", AIAA Paper 97-0393, Jan. 1997.

[4] Ramaswamy, M. A., "FMRL'S 6-Component Jet Rig Balance", FMRL's internal report, 1995, FAMU/FSU college of Engineering, Tallahassee, Florida. 

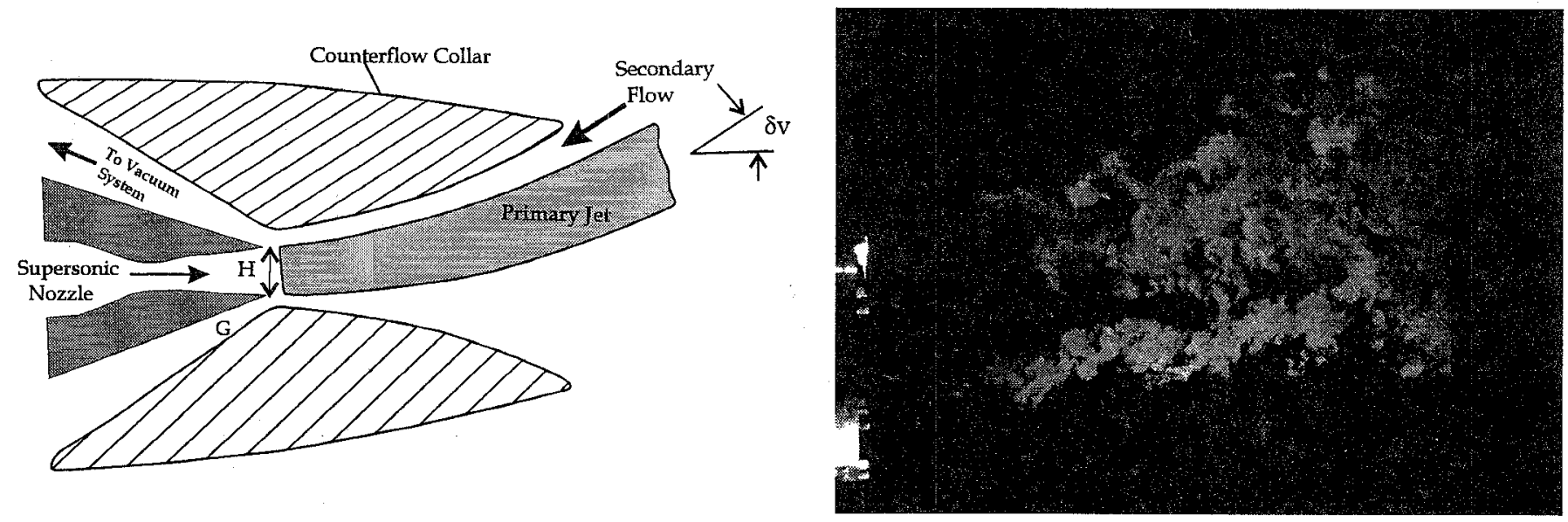

Fig. 1 - Schematic of CFTVC nozzle-collar assembly.

Fig. 2 - PLS image of a vectored axisymmetric (round) jet.

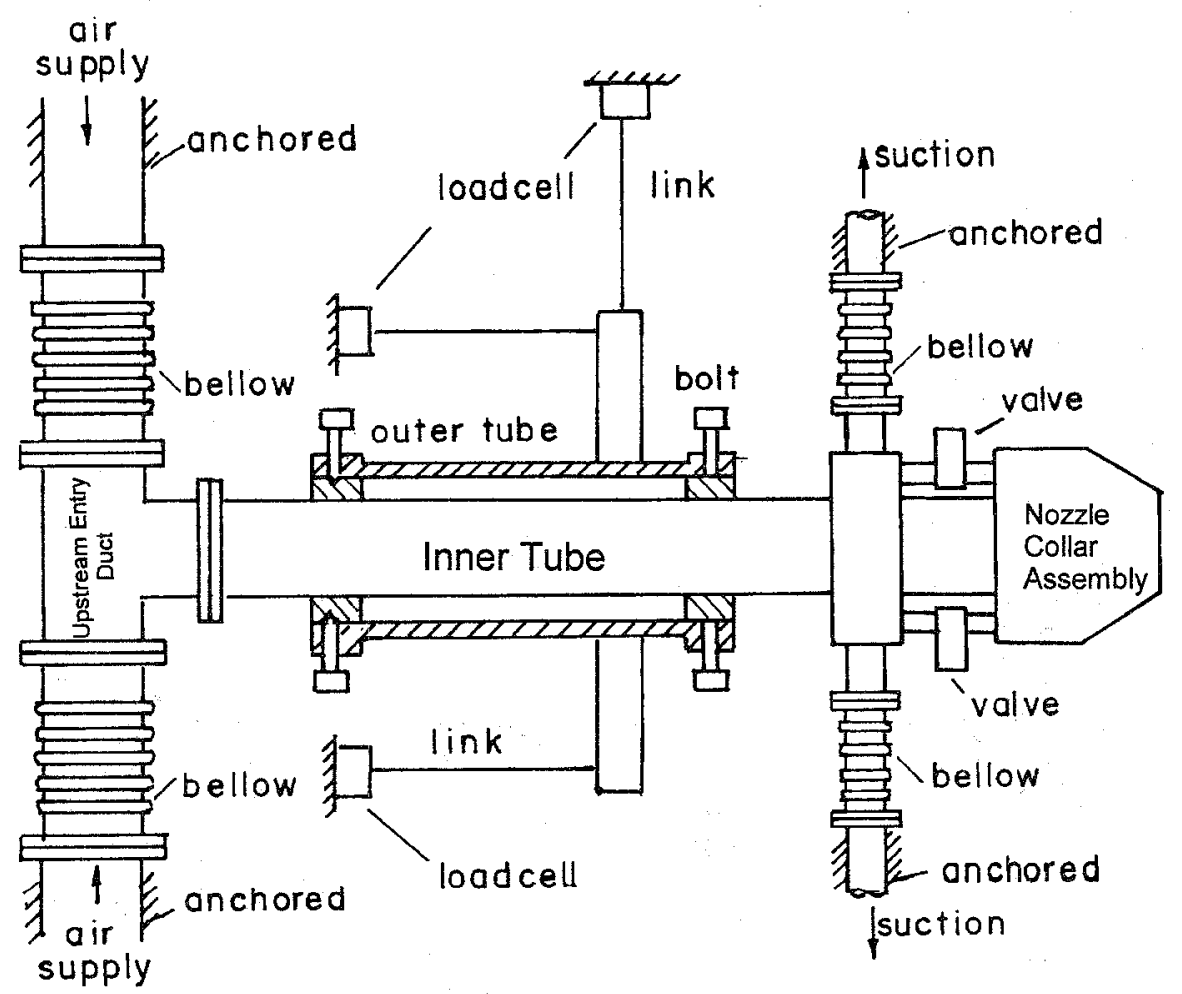

Fig. 3 - Schematic of the 3-component balance system. 


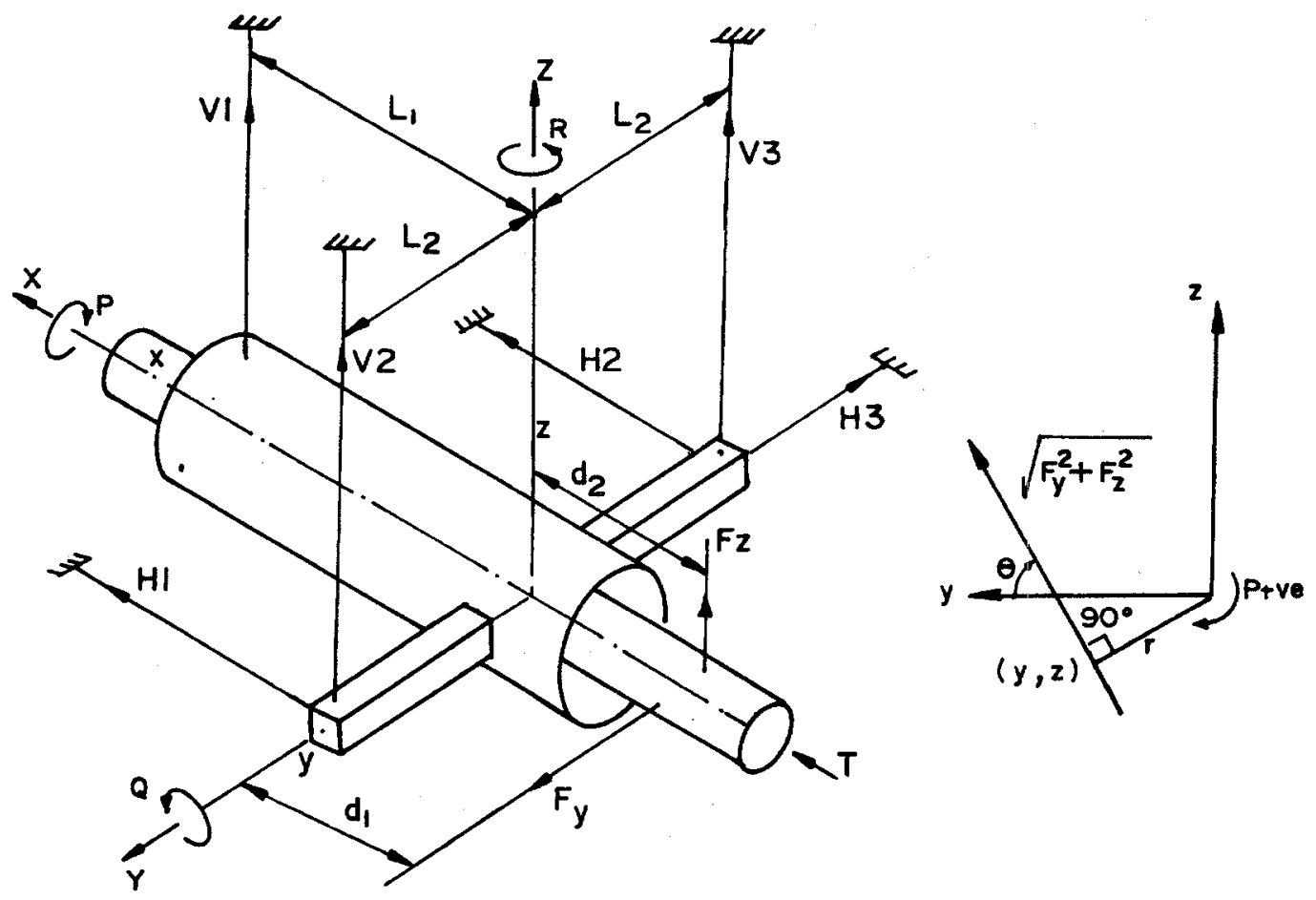

Fig. 4 - Conceptual design of the balance.

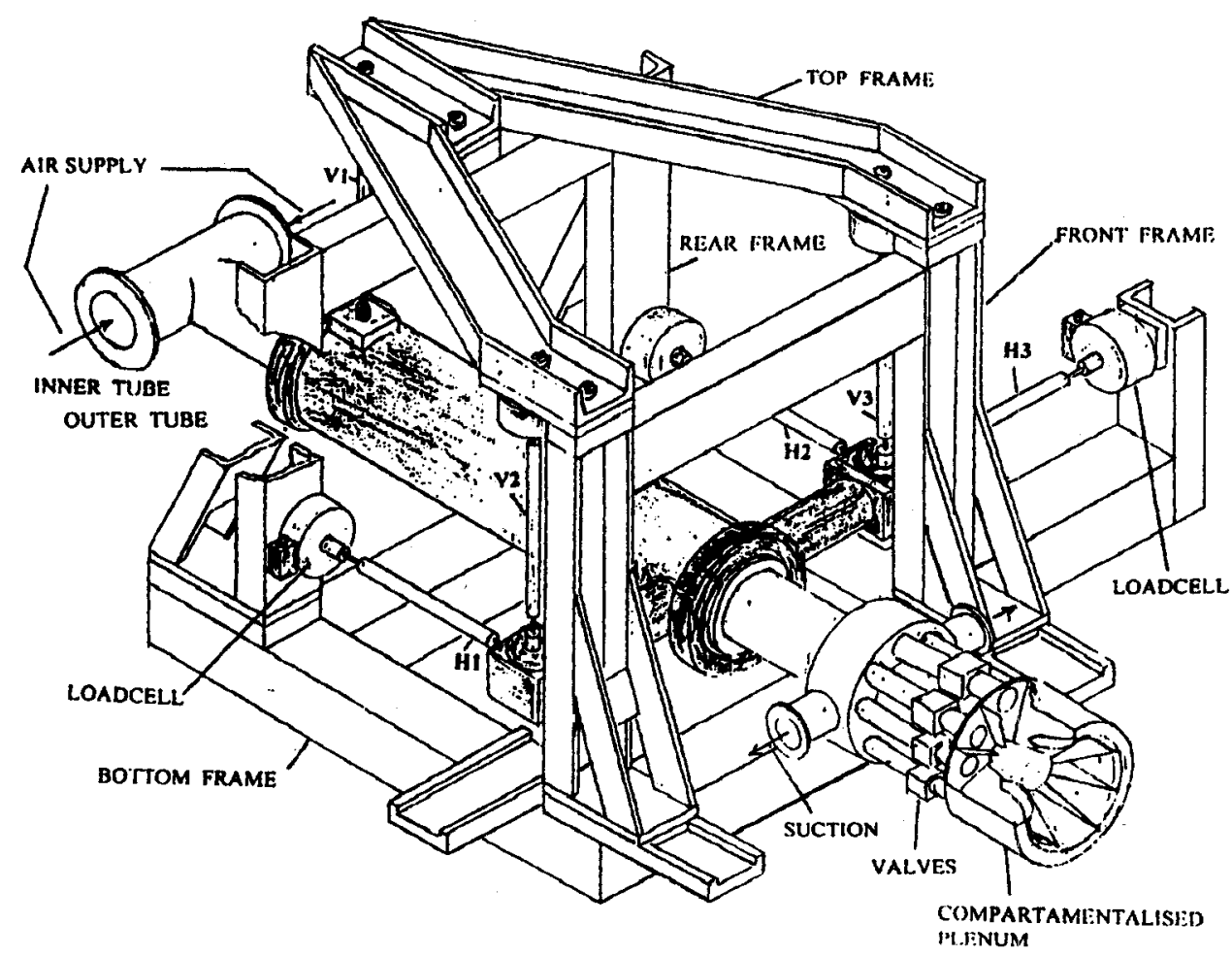

Fig. 5 - Isometric view of the balance. 

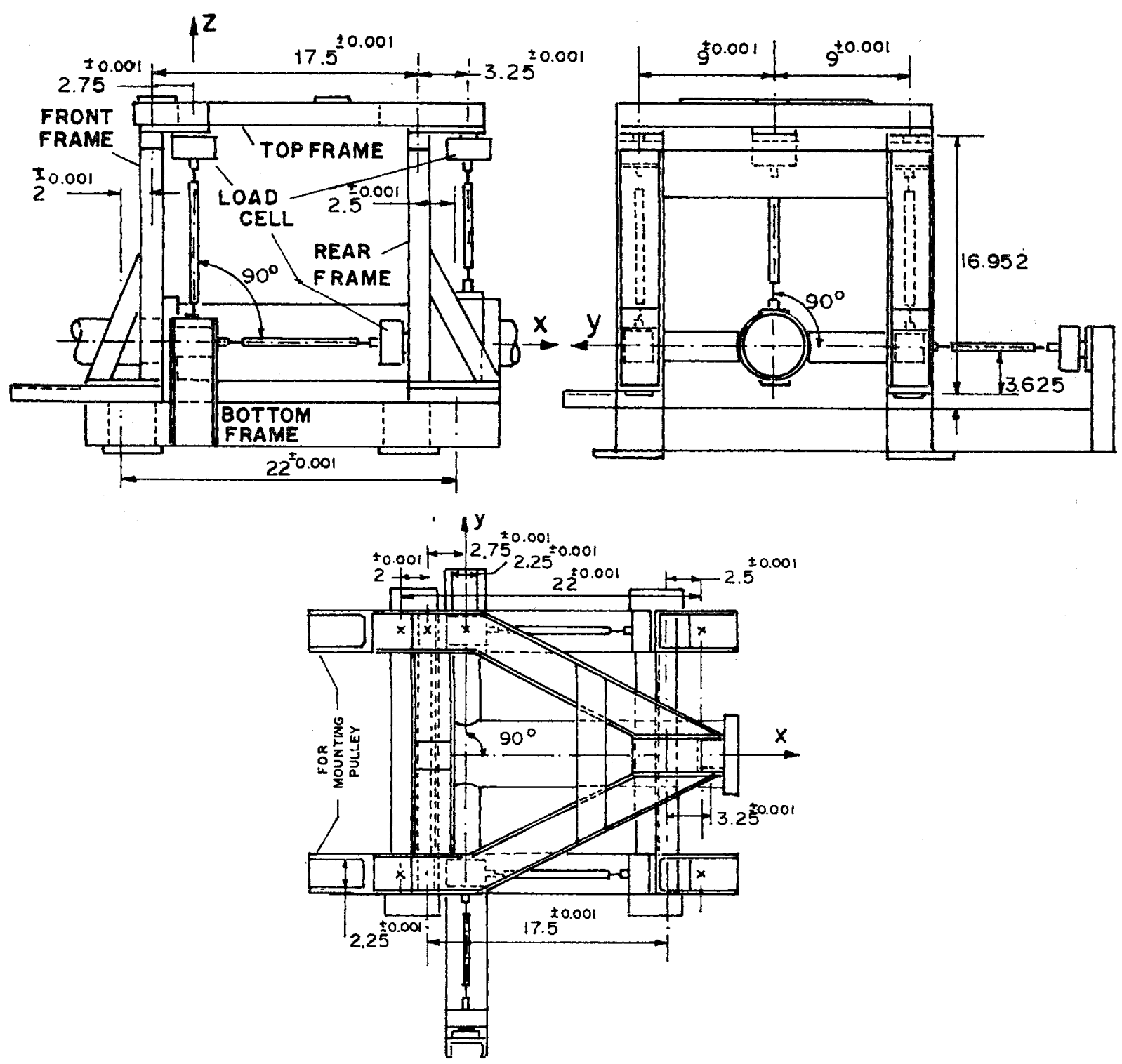

Fig. 6 - Orthogonal views of the balance (all dimensions are in inches).

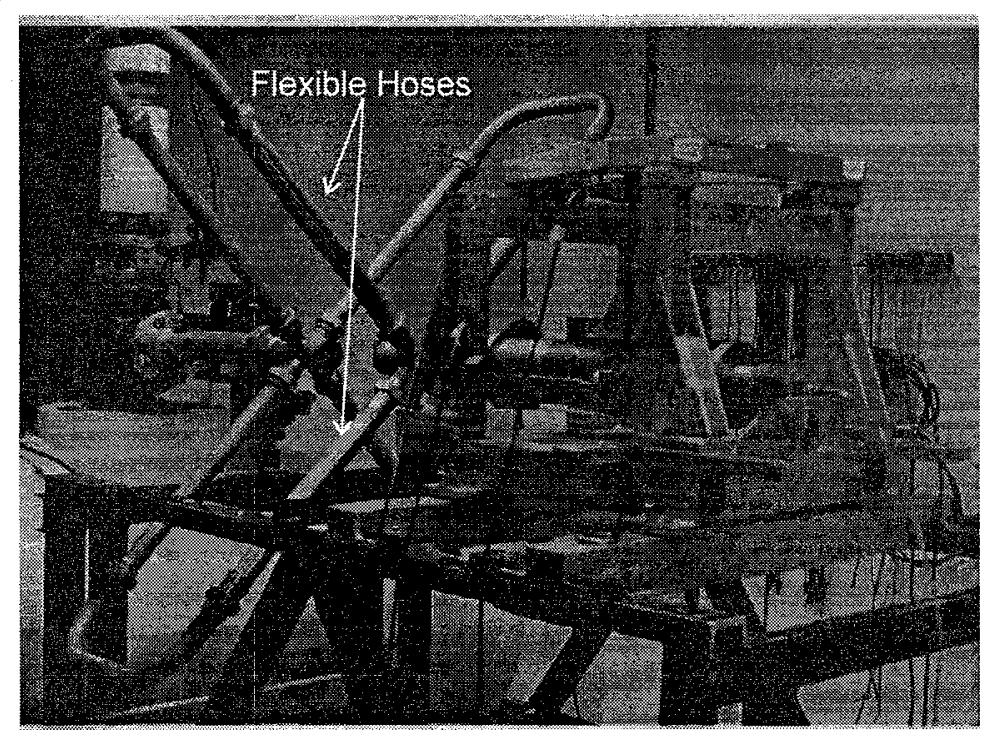

Fig. 7 - Image of the jet rig balance. 


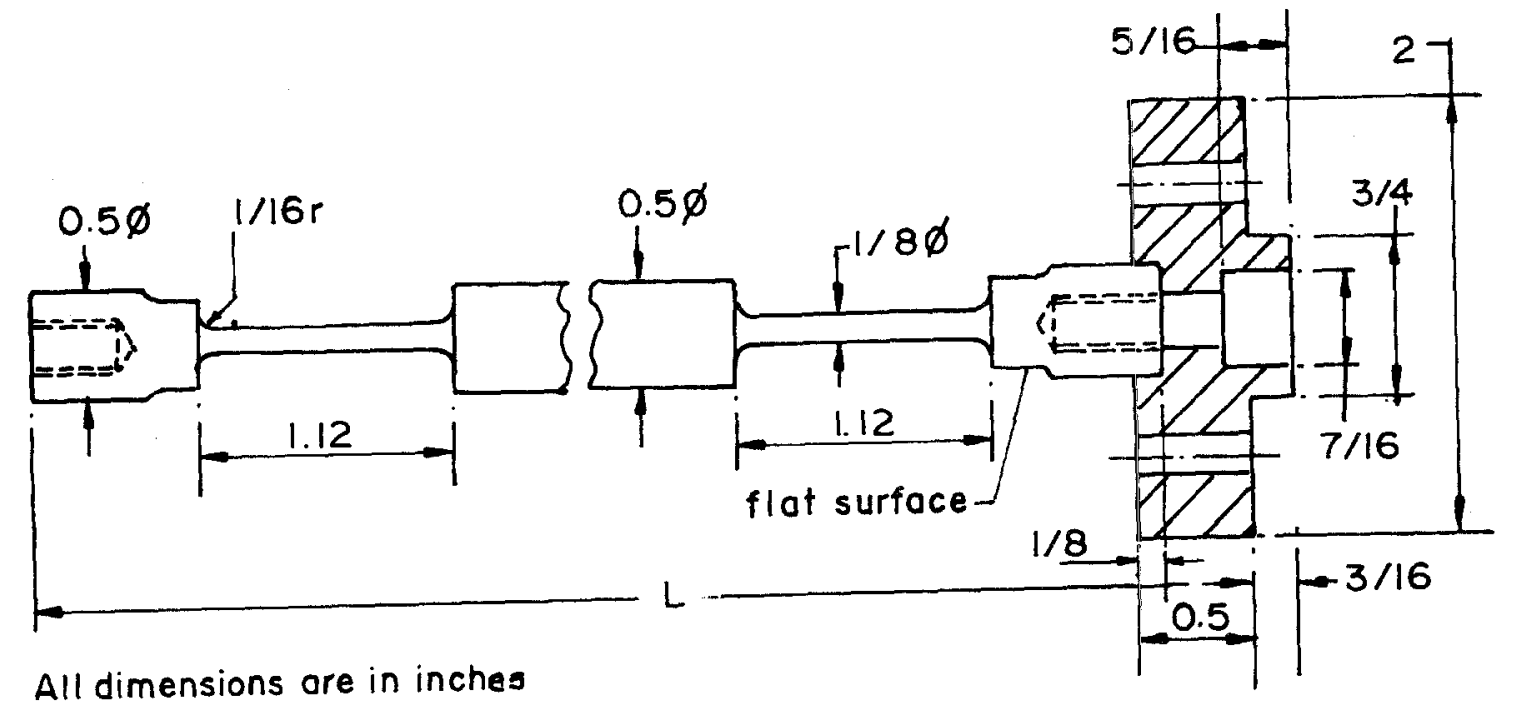

Fig. 8 - Detailed schematic of the short link.

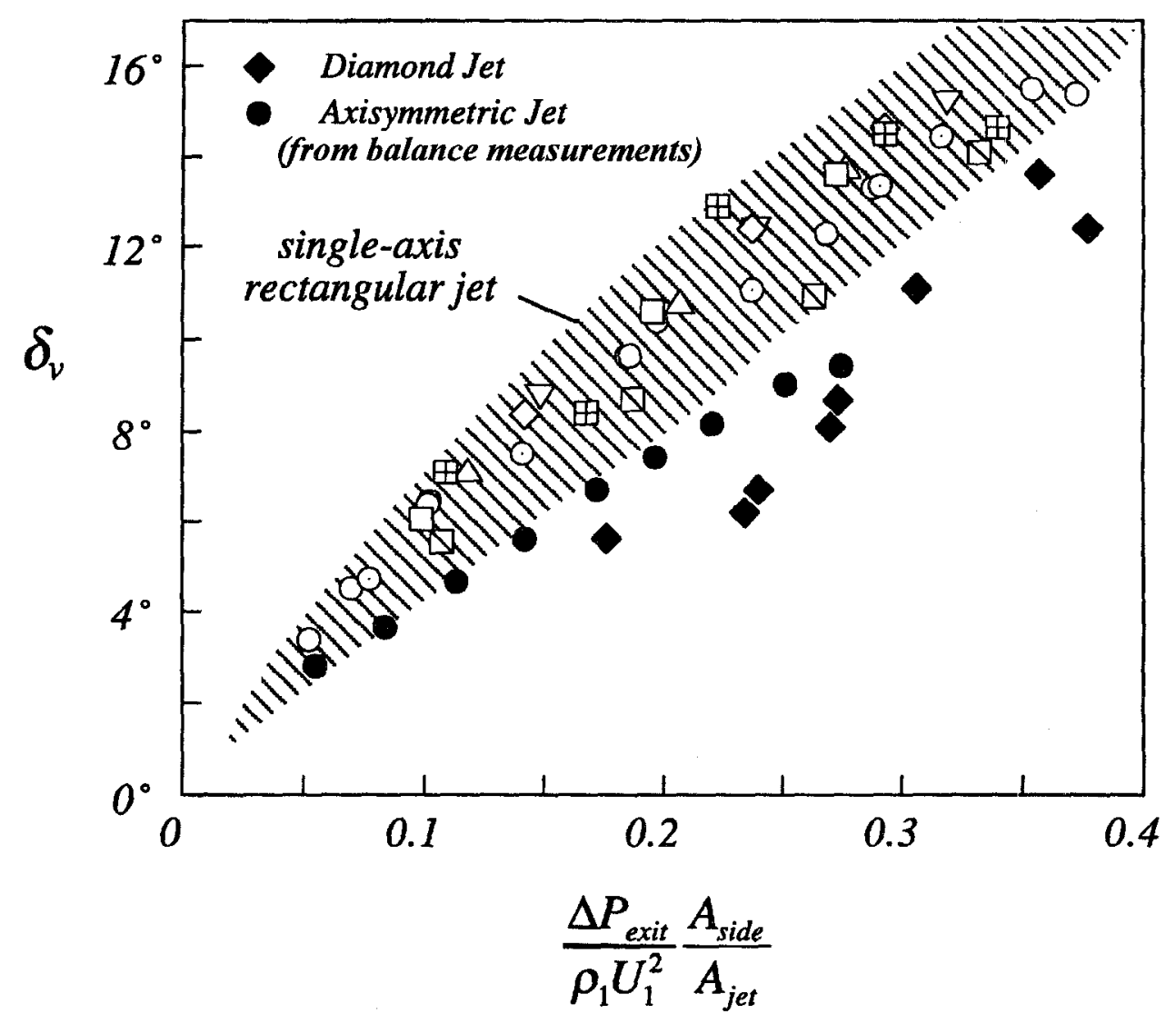

Fig. 9 - CFTVC performance for Mach 2 jets. 


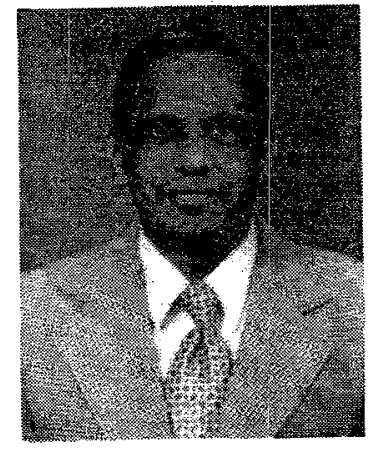

RAMASWAMY, M.A. received M.E. (Aero) from Indian Institute of Science (I.I.Sc.), Bangalore in 1958 and Ph.D. (Aero) from California Institute of Technology, Pasadena in 1971. He worked at I.I.SC. between 1958-1960. From 1960-1984, he worked in the National Aeronautical Laboratory, Bangalore, India and was involved in setting up the $4 \mathrm{ft}$. and $1 \mathrm{ft}$. high speed wind tunnel facilities and in directing the research and development activities of the Aerodynamics Division. Presently, he is a Professor at I.I.Sc. His interest are in high speed Aerodynamics, development of facilities and special test rigs. He is a Fellow of the National Academy of Science, India, the Indian National Academy of Engineering, the Aeronautical Society of India, and the Institute of Engineers, India. 


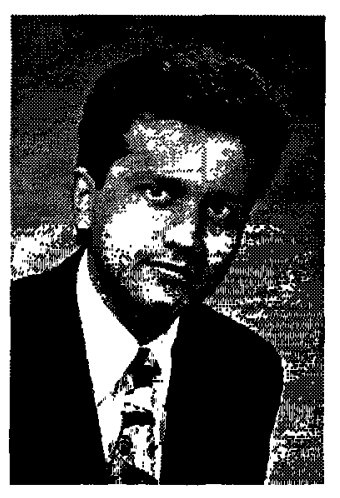

Dr. Farrukh S. Alvi is an assistant professor in the Department of Mechanical Engineering at the Florida A \& M University and Florida State University, Tallahassee. He received his $\mathrm{Ph}$. D. in Mechanical Engineering from the Pennsylvania State University, University Park, in 1992. His research interests include: compressible mixing, aeroacoustics of high speed jets, shock wave boundary layer interactions and optical diagnostics.

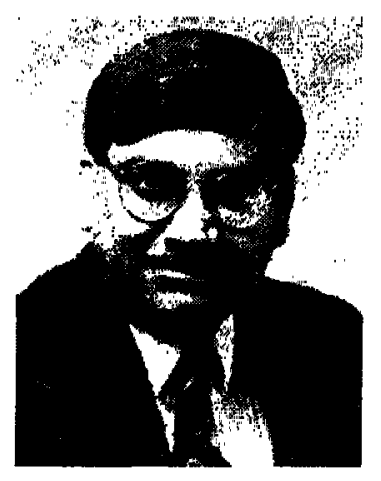

Dr. A. Krothapalli is the Don Fuqua Eminent Scholar, Professor, and Chairman of the Department of Mechanical Engineering at Florida A \& M University and Florida State University, Tallahassee. He obtained his $\mathrm{Ph}$. D. in Aeronautical Engineering from Stanford university in 1979. He has been on the faculties of Aerospace Engineering at the University of Oklahoma (1979-1980) and the Department of Aeronautics and Astronautics at Stanford University (1981-1983). His research interests include: aeroacoustics, aerodynamics, jets, wakes and optical diagnostics. 